\title{
Application of dried blood spots to determine vitamin D status in a large nutritional study with unsupervised sampling: the Food4Me project
}

Ulrich Hoeller $^{1 *}$, Manuela Baur ${ }^{1}$, Franz F. Roos ${ }^{1}$, Lorraine Brennan ${ }^{2}$, Hannelore Daniel ${ }^{3}$, Rosalind Fallaize ${ }^{4}$, Hannah Forster ${ }^{2}$, Eileen R. Gibney ${ }^{2}$, Mike Gibney ${ }^{2}$, Magdalena Godlewska ${ }^{5}$, Kai Hartwig ${ }^{3}$, Silvia Kolossa ${ }^{3}$, Christina P. Lambrinou ${ }^{6}$, Katherine M. Livingstone ${ }^{7}$, Julie A. Lovegrove ${ }^{4}$, Anna L. Macready ${ }^{4}$, Yannis Manios ${ }^{6}$, Cyril F. M. Marsaux ${ }^{8}$, J. Alfredo Martinez ${ }^{9,10}$, Carlos Celis-Morales ${ }^{7}$, George Moschonis $^{6}$, Santiago Navas-Carretero ${ }^{9,10}$, Clare B. O’Donovan ${ }^{2}$, Rodrigo San-Cristobal ${ }^{9,10}$, Wim H. M. Saris ${ }^{8}$, Agnieszka Surwiłło ${ }^{5}$, Iwona Traczyk ${ }^{5}$, Lydia Tsirigoti ${ }^{6}$, Marianne C. Walsh ${ }^{2}$, Clara Woolhead ${ }^{2}$, John C. Mathers ${ }^{7}$ and Peter Weber ${ }^{1}$ on behalf of the Food4Me project

${ }^{1}$ DSM Nutritional Products, Analytical Research Centre and Human Nutrition \& Health, 4002 Basel, Switzerland

${ }^{2}$ UCD Institute of Food and Health, University College Dublin, Belfield, Dublin 4, Republic of Ireland

${ }^{3}$ ZIEL Research Center of Nutrition and Food Sciences, Biochemistry Unit, Technische Universität München, 85435 Freising, Germany

${ }^{4}$ Hugh Sinclair Unit of Human Nutrition and Institute for Cardiovascular and Metabolic Research, University of Reading,

Reading RG6 GAP, UK

${ }^{5}$ National Food \& Nutrition Institute, Warsaw 02903, Poland

${ }^{6}$ Department of Nutrition and Dietetics, Harokopio University, Kallithea 17671, Athens, Greece

${ }^{7}$ Human Nutrition Research Centre, Institute of Cellular Medicine, Newcastle University, Newcastle Upon Tyne NE1 7RU, UK

${ }^{8}$ Department of Human Biology, NUTRIM School of Nutrition and Translational Research in Metabolism, Maastricht University

Medical Centre, 6229 HX Maastricht, The Netherlands

${ }^{9}$ Department of Nutrition, Food Science and Physiology, University of Navarra, 31008 Pamplona, Spain

${ }^{10}$ CIBER Fisiopatología Obesidad y Nutrición (CIBERobn), Instituto de Salud Carlos III, Madrid, Spain

(Submitted 22 June 2015 - Final revision received 28 September 2015 - Accepted 1 October 2015 - First published online 9 November 2015)

\section{Abstract}

An efficient and robust method to measure vitamin $\mathrm{D}$ (25-hydroxy vitamin $\mathrm{D}_{3}\left(25(\mathrm{OH}) \mathrm{D}_{3}\right)$ and 25 -hydroxy vitamin $\mathrm{D}_{2}$ in dried blood spots (DBS) has been developed and applied in the pan-European multi-centre, internet-based, personalised nutrition intervention study Food4Me. The method includes calibration with blood containing endogenous $25(\mathrm{OH}) \mathrm{D}_{3}$, spotted as DBS and corrected for haematocrit content. The methodology was validated following international standards. The performance characteristics did not reach those of the current gold standard liquid chromatography-MS/MS in plasma for all parameters, but were found to be very suitable for status-level determination under field conditions. DBS sample quality was very high, and 3778 measurements of $25(\mathrm{OH}) \mathrm{D}_{3}$ were obtained from 1465 participants. The study centre and the season within the study centre were very good predictors of $25(\mathrm{OH}) \mathrm{D}_{3}$ levels $(P<0 \cdot 001$ for each case). Seasonal effects were modelled by fitting a sine function with a minimum $25(\mathrm{OH}) \mathrm{D}_{3}$ level on 20 January and a maximum on 21 July. The seasonal amplitude varied from centre to centre. The largest difference between winter and summer levels was found in Germany and the smallest in Poland. The model was cross-validated to determine the consistency of the predictions and the performance of the DBS method. The Pearson's correlation between the measured values and the predicted values was $r 0 \cdot 65$, and the sD of their differences was $21.2 \mathrm{nmol} / \mathrm{l}$. This includes the analytical variation and the biological variation within subjects. Overall, DBS obtained by unsupervised sampling of the participants at home was a viable methodology for obtaining vitamin D status information in a large nutritional study.

Key words: Vitamin D status: Dried blood spot sampling: Nutrition intervention study Food4Me: Analytical methods

Abbreviations: $25(\mathrm{OH}) \mathrm{D}_{2}$, 25-Hydroxy vitamin $\mathrm{D}_{2} ; 25(\mathrm{OH}) \mathrm{D}_{3}$, 25-hydroxy vitamin $\mathrm{D}_{3}$; DBS, dried blood spots; LOD, limit of detection; LOQ, limit of quantitation; $\mathrm{MeOH}$, methanol; RT, room temperature.

*Corresponding author: U. Hoeller, email ulrich.hoeller@dsm.com 
Dried blood spotting (DBS) is a minimally invasive technique to obtain blood samples on cards of filter paper for biochemical or genetic analysis. A small prick in a fingertip is enough to produce a drop of blood suitable for sampling. This approach has the advantages of being considerably less invasive than venous blood sampling, does not require healthcare professionals and can be performed by most people on themselves after relatively little training. In addition, if the analyte is stable in dried whole blood, samples can be transported at room temperature (RT) by regular mail. Infrastructure for collecting venous blood samples, separation of plasma and storage and shipment of frozen samples is no longer required. In practice, blood sampling can be carried out anywhere and at any time. This offers significant benefits, especially for nutrient status determination in populations at remote locations or at home ${ }^{(1,2)}$

These advantages make the methodology ideal for use in an internet-based intervention such as the Food4Me study, where all data were self-reported and biological samples were collected remotely by the participants ${ }^{(3)}$. To date, the Food $4 \mathrm{Me}$ study is the largest multi-centre, internet-based, personalised nutrition intervention aiming to compare the effectiveness of three levels of personalised nutrition (based on dietary, phenotypic and genotypic data) on behavioural changes (diet and physical activity) and health outcomes (blood metabolites and obesity-related anthropometrics) ${ }^{(3)}$. The DBS technique was first introduced for diagnosing phenylketonuria in newborns by measuring whole-blood phenylalanine concentration ${ }^{(4)}$, and has since been applied to multiple metabolites ${ }^{(1,5)}$. A critical success factor for assessment of many vitamins and other micronutrients in DBS is their stability on the sampling cards. The compounds need to be stable in the presence of $\mathrm{O}_{2}$ at ambient temperature for at least the initial drying time and shipment time to the laboratory. In the case of the Food4Me study, this period was about 1 week. Stability can be increased by adding reagents such as antioxidants to the sampling cards. However, this approach has limitations due to safety concerns as the impregnated card comes in close contact with the pricked fingertip. To date, DBS assays reported for micronutrients include the vitamins $\mathrm{A}^{(6,7)}, \mathrm{B}_{12}{ }^{(8)}, \mathrm{D}^{(9-13)}, \mathrm{K}^{(14)}$ and folate ${ }^{(15)}$. However, only the assays for vitamins $\mathrm{A}$ and $\mathrm{D}$ appear to be used frequently. Although vitamin A was reported to be somewhat unstable with a loss of $>10 \%$ over the 1 st week ${ }^{(16)}$, no stability issues have been reported for vitamin $\mathrm{D}$ (25-hydroxy vitamin $\mathrm{D}_{3}\left(25(\mathrm{OH}) \mathrm{D}_{3}\right)$ ). An explanation for the particular stability of this vitamin may be the presence of a specific vitamin D-binding protein in blood that (together with serum albumin) binds $>99 \%$ of the circulating 25-hydroxy vitamin $\mathrm{D}^{(17,18)}$, thereby stabilising the vitamin on the DBS cards. Vitamin D assays based on DBS have been reported by three independent research groups viz., Eyles et $a l^{(9)}$ and Newman et al. ${ }^{(11)}$ and by Higashi et $a l^{(12)}$. Although all methods focus on $25(\mathrm{OH}) \mathrm{D}_{3}$, some procedures also include related metabolites, including 25-hydroxy vitamin $\mathrm{D}_{2}\left(25(\mathrm{OH}) \mathrm{D}_{2}\right)$ and 3-epi-25-hydroxy vitamin $\mathrm{D}_{3}$. The main differences between the assays are in the detection and calibration methods used and the extent of validation data that have been published. To date, all methods have been based on reversed-phase liquid chromatography (LC)-MS/MS with electrospray ionisation $\left(\mathrm{ESI}^{+}\right)$ detection. Eyles and Higashi derivatised the analytes with a $\mathrm{N}$-containing Diels-Alder reagent (4-phenyl-1,2,4-triazoline-3, 5-dione), which increases the sensitivity during MS-detection significantly, but has two drawbacks: it introduces an additional timeconsuming step during sample preparation, and the derivatisation leads to formation of two stereoisomers that complicate chromatography. The method by Newman et al. ${ }^{(11)}$ does not use analyte derivatisation, but starts with four large punches of filter paper that limit automated handling in small vials during sample preparation. A later publication from the same group indicated that the method has been modified and now also includes derivatisation of the analytes $^{(13)}$. However, this approach requires by far the largest sample aliquots, that is, four 6-mm-diameter punches.

In general, calibration of DBS analysis is difficult due to the absence of blank samples in the case of endogenous analytes, and the fact that current reference analytics is performed using plasma or serum samples and not (dried) blood. All existing methods for vitamin D analysis share a quite tedious calibration approach using spiked samples of blood, vitamin D-depleted blood or plasma for calibration.

The aim of the present study was to develop a novel, quicker assay for $25(\mathrm{OH}) \mathrm{D}_{3}$ and $25(\mathrm{OH}) \mathrm{D}_{2}$ from DBS without chemical derivatisation to cope with the expected large sample numbers from the Food4Me study, which might have utility for future large studies and surveys. We focused particularly on improving the calibration methodology to allow direct comparison of the results with published vitamin D status data, which are derived typically from measurements using plasma. Aspects of method development are reported, together with method validation and performance data. The Food4Me study did not include venous blood sampling, which precluded direct comparison of the results obtained from unsupervised collection of DBS by the participants at home with a reference method. Quality measures including spot quality and consistency of status levels of each participant measured at each time point have been assessed. The vitamin D status results were correlated with variables such as seasonality and research centre and were compared with literature data.

\section{Methods}

Study design and participants ${ }^{(3)}$

The Food4Me intervention study was designed as a panEuropean randomised controlled trial (RCT) to determine whether providing personalised dietary advice leads to greater improvements in eating patterns and health outcomes compared with a conventional population-based general guidelines approach. Seven research centres in seven European countries participated with more than 220 participants from each centre. DBS samples were collected and analysed at three time points: at baseline, after 3 and 6 months of intervention, respectively. Details of the study design and baseline characteristics of the participants have been published elsewhere $^{(3)}$. The seven participating study centres were located in Munich (Germany), Athens (Greece), Dublin (Ireland), Maastricht (The Netherlands), Warsaw (Poland), Navarra (Spain) and Reading (UK), and recruitment was carried out countrywide. A total of 5562 participants (65\% females) 
were screened online over a 12-month period between August 2012 and August 2013 and consented to participate. Of these, 1607 (28.9\%) were recruited to the RCT. Participants aged 18-79 (mean 39.8 (SD 13.1)) years were included in the study, of whom $60.9 \%$ ( $n$ 980) were women and $96.7 \%$ were from whiteEuropean background. The mean BMI of all the participants was $25.5(\mathrm{sD} 5 \cdot 2) \mathrm{kg} / \mathrm{m}^{2}$, and $44.8 \%(n 721)$ of the participants were overweight or obese $\left(\mathrm{BMI} \geq 25.0 \mathrm{~kg} / \mathrm{m}^{2}\right)$. This study was conducted according to the guidelines laid down in the Declaration of Helsinki, and all the procedures involving human subjects were approved by the ethics committees of the participating centres. Written or online informed consent was obtained from all the participants. Further details on study design and participant characteristics can be found in the study by Celis-Morales et al. ${ }^{(3,19)}$.

\section{Samples and sampling protocol}

Finger-prick blood samples were collected by participants themselves using a collection pack with two cards, one provided by Vitas Ltd, and one by DSM. Before spotting blood, cards for vitamin D analysis (Whatman Protein Saver 903 Card; GE Healthcare) were pre-treated with $1 \%$ of 2,6-di-tert-butyl-4-methylphenol (BHT) dissolved in methanol $(\mathrm{MeOH}) ; 30 \mu \mathrm{l}$ of $1 \% \mathrm{BHT}$ in $\mathrm{MeOH}$ were pipetted to each circle on the card and allowed to dry for at least $30 \mathrm{~min}$ at RT. These pre-treated cards were packed in an airtight $\mathrm{Al}$ bag (Whatman Foil Bags, item no. 10534321; Whatman Inc.) with a drying agent (Sorb-it, item no. 10548234; Süd-Chemie) and stored at RT until use. To help with blood collection, participants had access to an online video demonstration with written instructions and frequently asked questions in the language of recruitment. For the finger pricks, 2-0-mm contact-activated lancets (BD Microtainer; Becton, Dickinson and Company) were used. Each participant was asked to fill the two DBS cards each with five spots at each collection time point. This is the approximate equivalent of five drops of blood or $250 \mu \mathrm{l}$ of blood/card. When the ten blood spots were filled, participants were instructed to let the cards dry at RT for at least $2 \mathrm{~h}$, but not longer than $4 \mathrm{~h}$, before samples were put back into the $\mathrm{Al}$ bags and returned by mail to the corresponding recruiting centre. The centres verified the content of the bags, and shipped one bag containing the DBS card to DSM (DSM Nutritional Products Ltd) for measurements of vitamin D $\left(25(\mathrm{OH}) \mathrm{D}_{2}\right.$ and $\left.25(\mathrm{OH}) \mathrm{D}_{3}\right)$. Although the shipments carried out done at ambient temperature, the closed bags were stored at the centres and at DSM at nominal $-20^{\circ} \mathrm{C}$.

Calibration was carried out using whole-blood samples received from blood donors of the 'Blutspendezentrum SRK beider Basel' (Blood Donation Centre at Basel Hospital), including haematocrit values for each sample. The calibration samples ( $n$ 15) had a mean haematocrit content of $44.2 \%$ (range 38.7-49.3\%). Donors were twelve females and three males with an average age of 49 years. The calibration samples were prepared as follows: blood aliquots of $50 \mu \mathrm{l}$ were pipetted onto a card and allowed to dry for $2-4 \mathrm{~h}$ at RT, avoiding exposure to direct sunlight; the cards were then transferred into $\mathrm{Al}$ foil bags with a desiccant inside and then stored in a freezer at nominal $-20^{\circ} \mathrm{C}$. Calibration samples were used for 3-6 months. As quality control, DBS samples with independently determined $25(\mathrm{OH}) \mathrm{D}_{3}$ content were analysed within each analytical run.

\section{Reagents and instruments}

25-Hydroxycholecalciferol monohydrate (25-OH-D 3 monohydrate) was obtained from Dr Ehrenstorfer; 25-hydroxyergocalciferol $\left(25(\mathrm{OH}) \mathrm{D}_{2}\right)$ and SDS ( $\left.\geq 99 \%\right)$ were supplied by Sigma-Aldrich; 26,26,26,27,27,27-hexadeutero-25-hydroxycholecalciferol (25(OH) $\mathrm{D}_{3}$-d6) was supplied by Medical Isotopes; 2,6-di-tert-butyl-4methylphenol ( $\geq 99 \%$, BHT), acetonitrile (gradient grade), formic acid (Suprapur), toluene and $\mathrm{MeOH}$ were from Merck; and MS-grade water was prepared using a Milli-Q instrument (Merck Millipore). Eppendorf tubes were centrifuged using a $5417 \mathrm{R}$ model centrifuge (Vaudaux-Eppendorf), and evaporation was carried out using a Cyclone (Prolab). Analyte separation was performed using an Agilent 1260 HPLC with auto sampler, two binary pumps and column oven coupled with an AB Sciex Qtrap 5500 MS/MS-System with atmospheric pressure photoionisation (APPI source).

\section{Assay}

Sample preparation. Before analysis, the samples were assessed to check whether they met the quality criteria (Fig. 1): spot size (circle filled), thoroughly soaked (observed from the back) and one application of blood (not composed of many small spots). Two punches (inner diameter of $3.175 \mathrm{~mm}$ ) were taken out of the card and placed into a 2-ml Eppendorf tube. After adding $100 \mu \mathrm{l}$ of $0.1 \%$ SDS solution and $20 \mu \mathrm{l}$ of internal standard (ISTD, $25,25,25,26,26,26$-hexa-deutero- $25(\mathrm{OH}) \mathrm{D}_{3}, 25 \mathrm{ng} / \mathrm{ml}$ in $\left.\mathrm{MeOH}\right)$,

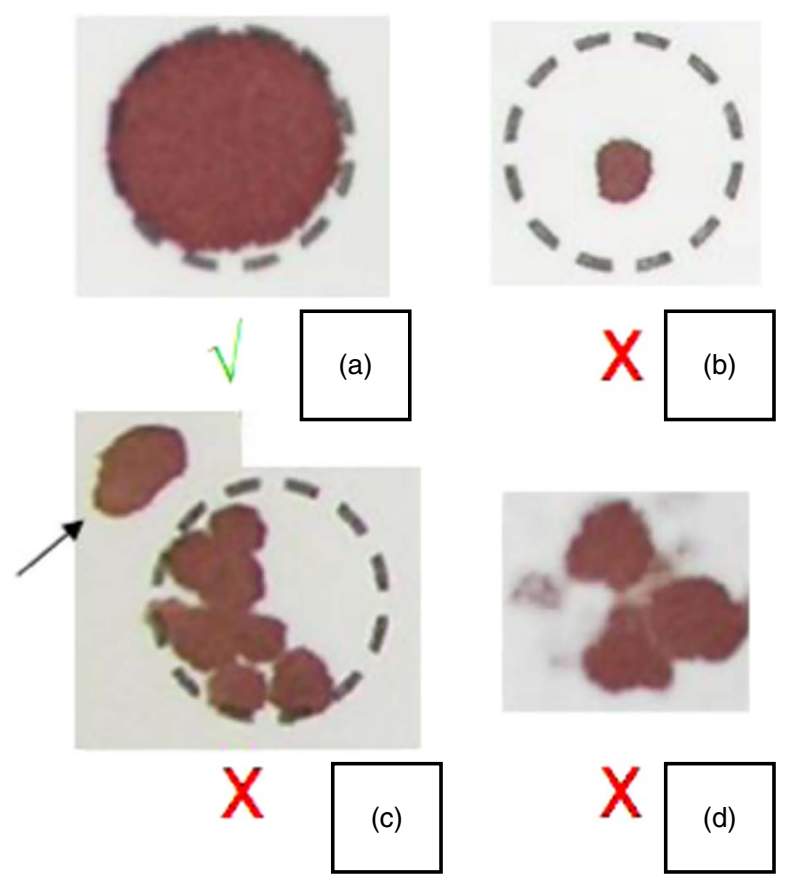

Fig. 1. Quality control criteria for dried blood spots from the Food4Me study. (a) Spot suitable for analysis. (b-d): Spots not suitable for analysis due to (b) small spot size not filling the circle, (c) multiple application of too small spots, including spots outside the circle, (d) multiple application of small spots and no thorough soaking of the paper (view from the back). 
the tubes were shaken at $40^{\circ} \mathrm{C}$ for $30 \mathrm{~min}$. Subsequently, $400 \mu \mathrm{l}$ of acetonitrile was added and the shaking continued at RT for $5 \mathrm{~min}$ (260/min, IKA shaker). Following this, the tubes were centrifuged at $20000 \boldsymbol{g}$ for $5 \mathrm{~min}$, and the supernatant was transferred into a new 2-ml Eppendorf tube. The solvent was evaporated to dryness under vacuum (Cyclone), and the residue was reconstituted with 50- $\mu \mathrm{l}$ injection solvent (MeOH-water, 70:30) and transferred into a micro vial for analysis.

Chromatography and detection. Chromatography was performed using an Ascentis Express C18 column (Supelco), $7.5 \times 2.1 \mathrm{~mm}, 2.7 \mu \mathrm{m}$, with a guard column using the following gradients: $0 \mathrm{~min}$, flow $600 \mu \mathrm{l} / \mathrm{min}, 15 \% \mathrm{~A} ; 1.8 \mathrm{~min}, 600 \mu \mathrm{l} / \mathrm{min}$, $0 \% \mathrm{~A} ; 1.9 \mathrm{~min}, 1000 \mu \mathrm{l} / \mathrm{min}, 0 \% \mathrm{~A} ; 3.7 \mathrm{~min}, 1000 \mu \mathrm{l} / \mathrm{min}, 0 \% \mathrm{~A}$; $3.8 \mathrm{~min}, 1000 \mu \mathrm{l} / \mathrm{min}, 15 \% \mathrm{~A} ; 5.8 \mathrm{~min}, 1000 \mu \mathrm{l} / \mathrm{min}, 15 \% \mathrm{~A} ; 5.9 \mathrm{~min}$, $600 \mu \mathrm{l} / \mathrm{min}, 15 \% \mathrm{~A}$; and $6.0 \mathrm{~min}, 600 \mu \mathrm{l} / \mathrm{min}, 15 \% \mathrm{~A}$. Mobile phase A consisted of water containing $0.05 \%$ formic acid; mobile phase $\mathrm{B}$ consisted of $\mathrm{MeOH}$-acetonitrile (80:20, v/v) containing $0.05 \%$ formic acid. Samples were kept at $10^{\circ} \mathrm{C}$, the column temperature was $30^{\circ} \mathrm{C}$ and the injection volume was $10 \mu \mathrm{l}$. Dopant for APPI detection was toluene added post-column at $100 \mu \mathrm{l} / \mathrm{min}$. Typical retention times were $1.55 \mathrm{~min}\left(25(\mathrm{OH}) \mathrm{D}_{3}\right.$ and ISTD) and $1.71 \min \left(25(\mathrm{OH}) \mathrm{D}_{2}\right)$.

Detection was carried out using an AB Sciex 5500 Qtrap instrument with APPI positive mode and MRM scan type at unit resolution.

Parameter table: CUR 20; CAD Medium (9); IS 775; TEM 320; GS1 75; GS2 50; EP 10.

25(OH)D 3 , 383.3 Q1 Mass (Da), 211.3 Q3 Mass (Da), 50 Dwell (ms), $66 \mathrm{DP}, 35 \mathrm{CE}, 8 \mathrm{CXP} ; 25(\mathrm{OH}) \mathrm{D}_{3}$ qualifier, 383.3 Q1 Mass (Da), 257.2 Q3 Mass (Da), 50 Dwell (ms), 91 DP, 21 CE, 12 CXP; ISTD 25(OH)D $-\mathrm{d} 6,389.3$ Q1 Mass (Da), 211.3 Q3 Mass (Da), 50 Dwell (ms), 51 DP, $41 \mathrm{CE}, 8 \mathrm{CXP} ; 25(\mathrm{OH}) \mathrm{D}_{2}, 395 \cdot 2 \mathrm{Q} 1$ Mass (Da), 269.2 Q3 Mass (Da), 50 Dwell (ms), 46 DP, 29 CE, $12 \mathrm{CXP} ; 25(\mathrm{OH}) \mathrm{D}_{2}$ qualifier, 395.2 Q1 Mass (Da), 209.2 Q3 Mass (Da), 50 Dwell (ms), 56 DP, 39 CE, 10 CXP.

\section{Determination of nominal 25-hydroxy vitamin $D_{3}$ content} of calibration samples. An aliquot of each whole-blood sample spotted for calibration was used for preparation of plasma. The $25(\mathrm{OH}) \mathrm{D}_{3}$ content of this plasma was measured using an established reference method ${ }^{(20)}$. The haematocrit content of the whole blood used for calibration was measured at the blood donation centre. As the haematocrit contents of the study samples were unknown, an estimated mean value of $40 \%$ was used for calculation. This value was based on the rounded mean haematocrit content of the samples used for method development and validation. The following equation was used to normalise the calibration samples accordingly:

$\mathrm{c}\left(25(\mathrm{OH}) \mathrm{D}_{3}\right)_{\text {normalised }}=\mathrm{c}\left(25(\mathrm{OH}) \mathrm{D}_{3}\right)_{\text {measured }} \times \frac{(100-\text { haematocrit })}{60}$.

$\mathrm{c}\left(25(\mathrm{OH}) \mathrm{D}_{3}\right)_{\text {normalised }}$ concentration of $25(\mathrm{OH}) \mathrm{D}_{3} \quad(\mathrm{ng} / \mathrm{ml}$ plasma) in a calibration sample, normalised to $40 \%$ haematocrit content; $c\left(25(\mathrm{OH}) \mathrm{D}_{3}\right)_{\text {measured }}$ concentration of $25(\mathrm{OH}) \mathrm{D}_{3}$ ( $\mathrm{ng} / \mathrm{ml}$ plasma) measured with reference method in plasma samples obtained from whole blood used for calibration; haematocrit: haematocrit value (\%) for whole-blood sample obtained from Blutspendezentrum Basel.

For study samples, the resulting $25(\mathrm{OH}) \mathrm{D}_{3}$ concentration was then corrected for sex-specific mean haematocrit values of $41.5 \%$ for female and $46.5 \%$ for male participants by applying correction factors of 1.026 for females and 1.121 for males. This was based on information from the sex-specific reference ranges and means obtained from the seven clinical centres (U Hoeller et al., personal information).

\section{Performance criteria}

The method was validated based on the procedures described in the 'Guideline on bioanalytical method validation' of the European Medicines Agency ${ }^{(21)}$, taking into account specific requirements and recommendations for DBS analysis ${ }^{(22)}$. Selectivity was tested by comparing chromatograms from five samples of different donors with and without spike of either $25(\mathrm{OH}) \mathrm{D}_{3}-\mathrm{d} 6$ or $25(\mathrm{OH}) \mathrm{D}_{3}$ at $20 \mathrm{ng} / \mathrm{ml}$ blood. As the samples contained endogenous $25(\mathrm{OH}) \mathrm{D}_{3}$, area ratios of qualifier ion $\mathrm{m}: \mathrm{z}(383: 257)$ to quantifier ion $\mathrm{m}: \mathrm{z}$ (383:211) were calculated and used for the assessment of selectivity of the detection of $25(\mathrm{OH}) \mathrm{D}_{3}$. Carry over was assessed by measuring blank samples after analysis of high-content samples. No significant carry over was observed. Linearity was determined by analysis of calibration samples according to the method on 3 different $\mathrm{d}$. Linear regression of the analyte peak area ratio (analyte peak area $v$. ISTD peak area) against the nominal concentration was calculated. Differences between nominal concentrations and calculated concentrations were determined and expressed in percentage. Deviations of the measured values from nominal values should be $\leq 20 \%$ and at least two-thirds of the calibration levels at each day should meet these criteria. As calibration was performed with endogenous samples, only a limited concentration range could be tested: 32.5-120 nmol/1 calculated as plasma concentrations. The lower limit of quantification was set to $25 \mathrm{nmol} / \mathrm{l}$, as this represents the accepted cut-off for vitamin D deficiency ${ }^{(23)}$. Accurate determination of lower concentrations was not within the scope of this study. For assessing accuracy, samples of five different subjects were analysed. Before spotting the whole-blood samples, plasma was separated from a whole blood aliquot of each sample and analysed using a reference $\operatorname{method}^{(24)}$. Nominal DBS concentrations were calculated by correcting the plasma concentrations with the corresponding haematocrit values (nominal concentration $=$ plasma concentration $\times(100-$ haematocrit value)/100). DBS samples from each subject were analysed in 5-fold and compared with the nominal DBS concentration. Mean accuracy was calculated and reported as percentage of nominal DBS concentration value. As additional measure for accuracy and of the influence of varying haematocrit values in the calibration samples, we re-analysed calibration samples for which the $25(\mathrm{OH}) \mathrm{D}_{3}$ concentration values were assigned previously within a new set of calibration samples with independently assigned $25(\mathrm{OH}) \mathrm{D}_{3}$ concentration values. The mean haematocrit value of these samples was 42.0 (range 39.6-43.8)\%. To determine intra-day precision, the results from samples prepared for accuracy were used. To estimate inter-day precision, triplicates of each sample were analysed on 3 different $d$. Precision was reported as $\mathrm{CV}$ of the measured concentrations. 
For determination of stability in the auto sampler at $10^{\circ} \mathrm{C}$, extracts of incurred and spiked DSB samples were re-analysed after $60 \mathrm{~h}$. Long-term stability was tested by storing DBS samples for upto 6 months at nominal $-20^{\circ} \mathrm{C}$ and comparing the content analysed after the storage with initial values. There was good stability under both conditions.

\section{Statistical analysis}

Longitudinal linear mixed models were used to model $25(\mathrm{OH}) \mathrm{D}_{3}$ levels. Limit of detection (LOD) values in the $25(\mathrm{OH}) \mathrm{D}_{3}$ measurements were replaced by $12.5 \mathrm{nmol} / \mathrm{l}$ (four measurements, $0 \cdot 1 \%$ ) and lower limit of quantitation (LOQ) values were replaced by $16.25 \mathrm{nmol} / \mathrm{l} 25(\mathrm{OH}) \mathrm{D}_{3}$ (134 measurements, $3.5 \%$ ). Sensitivity analyses were carried out assuming $0 \mathrm{nmol} / \mathrm{l}$ for LOD and LOQ values, and $25 \mathrm{nmol} / \mathrm{l}$ for LOD and LOQ values, respectively. For the centre with by far the most LOQ values (Dublin), the model fits differed by $-4.0 \mathrm{nmol} / 1$ in winter, assuming $0 \mathrm{nmol} / 1$ for LOD and LOQ, and $+0.2 \mathrm{nmol} / 1$ in summer. Assuming $25 \mathrm{nmol} / 1$ for LOQ and LOD values, the difference was $+2 \cdot 2 \mathrm{nmol} / 1$ in winter and $-0.1 \mathrm{nmol} / \mathrm{l}$ in summer. The absolute deviations for all other centres were $\leq 2.4 \mathrm{nmol} / 1$ in winter and $\leq 0.2 \mathrm{nmol} / 1$ in summer.

To model the seasonal variation at the study sites, the study centre and the interaction of study centre with the functions sin (sample year $\times 2 \times \pi$ ) and $\cos ($ sample year $\times 2 \times \pi$ ) were included as fixed effects, and the participants as a random effect. The 20 January was found to be the consensus date across all study centres when the $25(\mathrm{OH}) \mathrm{D}_{3}$ concentrations reached their nadir. To simplify the subsequent modelling and interpretation, a single normalised sine function was derived, which oscillated between -1.0 when the $25(\mathrm{OH}) \mathrm{D}_{3}$ concentration was at its lowest on the 20 January and $+1 \cdot 0$ when the $25(\mathrm{OH}) \mathrm{D}_{3}$ concentration was at its highest on the 21 July. It assumes synchronised timing of seasons across all study centres, but it differentiates mean levels and seasonal amplitudes by study centre. The function was coded as follows: standardised seasonal amplitude $=\mathrm{SSA}=\sin ($ (sample year $-20 / 365 \cdot 25-0 \cdot 25) \times 2 \times \pi)$. On the 20th of each month, this function had the following values: -1.0 in January, -0.9 in February, -0.5 in March, 0.0 in April, 0.5 in May, 0.9 in June, 1.0 in July, 0.9 in August, 0.5 in September, 0.0 in October, -0.5 in November and -0.9 in December. For a sample taken on the
30 June, the sample year would equal 2013.5, and the resulting SSA would be 0.94 . For each measurement, the corresponding SSA was thus calculated. The final model was then fitted using centre and the centre-SSA interaction as fixed effects and participant ID as random effect (presented in Table 2 and Fig. 2). Owing to the absence of a gold standard method, the biological and the analytical variability could not be separated in the present data set. The overall variation should thus be considered as a highly conservative estimate of the variation of the analytical method. $\mathrm{R}$ statistics software version 3.02 was used for all statistical analyses $^{(25)}$, and within the $\mathrm{R}$ statistics software the function lme () in the package nlme ${ }^{(26)}$ was used for the mixed model regressions.

\section{Results}

The assay described in the present study was developed for analysis of the large sample numbers from the Food4Me study. Calibration was performed using blood samples containing endogenous analyte content spotted on DBS cards. The nominal $25(\mathrm{OH}) \mathrm{D}_{3}$ concentration of these calibration samples was determined from plasma obtained from the same samples with an established reference method, corrected by the measured haematocrit values. Stability trials showed that these calibration samples could be used for at least 6 months if stored at nominal $-20^{\circ} \mathrm{C}$. The current method is equally applicable for determination of $25(\mathrm{OH}) \mathrm{D}_{2}$, but because substantial concentrations of $25(\mathrm{OH}) \mathrm{D}_{2}$ were not expected in the present study method validation focused on $25(\mathrm{OH}) \mathrm{D}_{3}$. An overview of the parameters validated and the results are given in Table 1 . The re-analysis of calibration samples within an independent calibration showed a good correlation between the originally assigned $25(\mathrm{OH}) \mathrm{D}_{3}$ concentrations and the concentrations determined from the independent calibration for these samples ( $n$ 5; $R$ 0.97). Overall, the DBS method showed results with slightly larger variations compared with current reference methodologies but within or close to the acceptance ranges of the guidelines. This led to specific characteristics of, for example, a rather small linear range of 32.5-120 nmol/l with deviation of upto $18 \%$ between measured and nominal values (guideline limit 15\%) and precision upto 13-14\% CV for interday and intra-day. Overall, it was concluded that the method

Table 1. Overview of the performance characteristics of the analytical method

\begin{tabular}{|c|c|c|}
\hline Validation parameters & Procedure & Result (representative values) \\
\hline Selectivity & Analysis of incurred and spiked samples & $\begin{array}{l}\text { Interference with } 25(\mathrm{OH}) \mathrm{D}_{3}-\mathrm{d} 6 \leq 0.4 \% \\
\text { Interference with } 25(\mathrm{OH}) \mathrm{D}_{3} \leq 14.2 \% \text { (quantifier:qualifier ratio) }\end{array}$ \\
\hline Carry over & Injection of blank after high-level spiked sample & $\begin{array}{l}\text { Carry over of } 25(\mathrm{OH}) \mathrm{D}_{3} \leq 1.6 \% \\
\text { Carry over of ISTD } \leq 0.12 \%\end{array}$ \\
\hline Linearity & Analysis of incurred samples & $\begin{array}{l}32.5-120 \mathrm{nmol} / \mathrm{l} \text { (as plasma concentration) } \\
\text { Deviation measured from nominal value } \leq 18 \% ; R^{2} \geq 0.95\end{array}$ \\
\hline Accuracy & Incurred sample compared with reference value & $80-95 \%$ \\
\hline Precision & Analysis of incurred sample & $\begin{array}{l}\text { Intra-day: } 5 \cdot 8-13.0 \% \mathrm{CV} \\
\text { Inter-day: } 8 \cdot 4-14.0 \% \mathrm{CV}\end{array}$ \\
\hline Recovery & Analysis of incurred and spiked samples & $74-101 \%$ \\
\hline Stability auto sampler $\left(10^{\circ} \mathrm{C}\right)$ & Comparison with starting values & $60 \mathrm{~h}$ at $10^{\circ} \mathrm{C}$; difference: $\leq 6.1 \%$ \\
\hline Stability long term & Comparison with starting values & 6 months at nominal $-20^{\circ} \mathrm{C}$; difference $\leq-14.1 \%$ \\
\hline
\end{tabular}

$25(\mathrm{OH}) \mathrm{D}_{3}$-d6, 26,26,26,27,27,27-Hexadeutero-25-hydroxycholecalciferol; ISTD, internal standard. 
All


Reading, UK

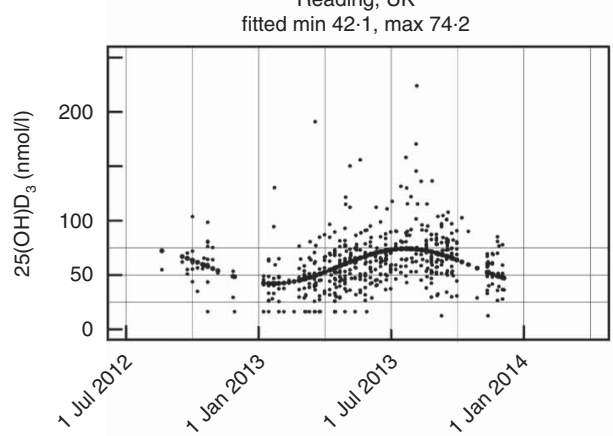

Athens, Greece

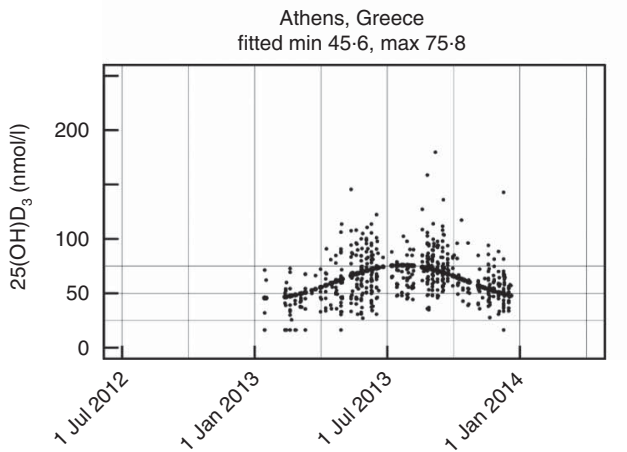

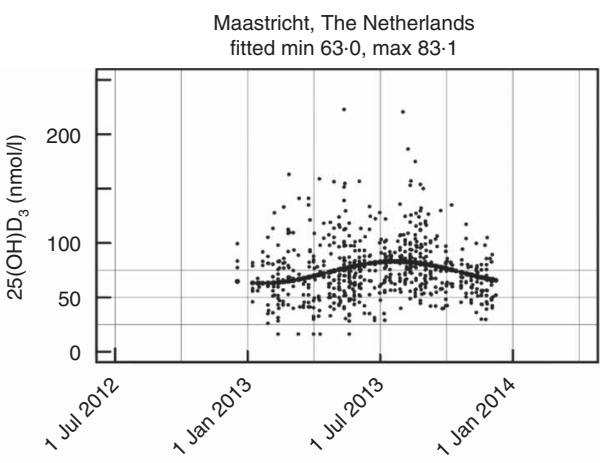

Dublin, Ireland

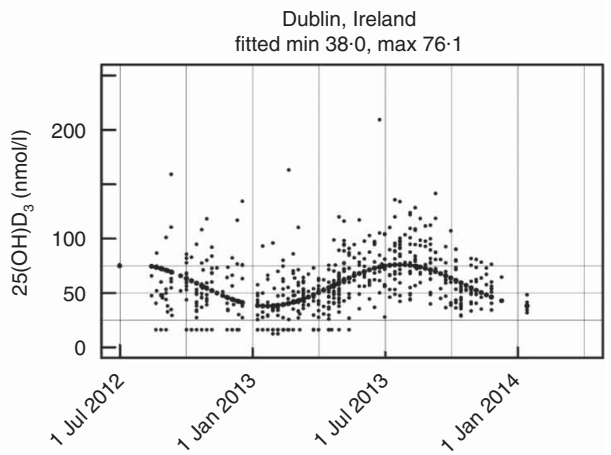

Warsaw, Poland

fitted $\min 50 \cdot 4, \max 67 \cdot 1$



Munich, Germany

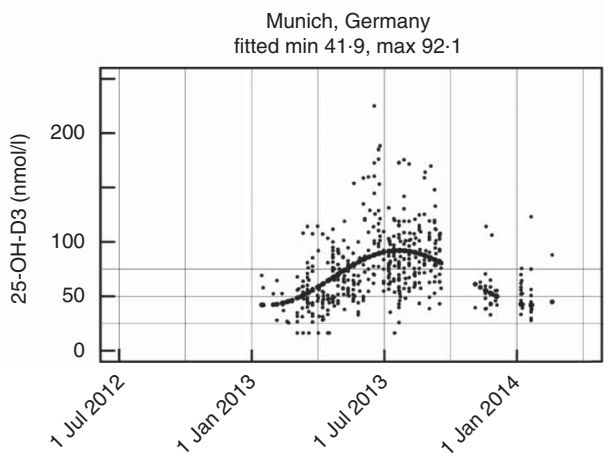

Fig. 2. Individual measurements for 25 -hydroxy vitamin $\mathrm{D}_{3}\left(25(\mathrm{OH}) \mathrm{D}_{3}\right)$ by research centre and sampling date, as well as seasonal regression by centre. The model included 3711 measurements from 1412 participants. The predictors were the centre and the interaction of each centre with the standardised seasonal amplitude (SSA). The SSA for this data set is a sine function reaching its minimum -1.0 on 20 January and its maximum +1.0 on 21 July, as explained in the statistical methods section. The participant ID was included as random effect. The fixed effect regression fits are visualised within each plot. The largest seasonal oscillations were observed in Germany $(92.1 \mathrm{nmol} / \mathrm{l}$ in summer v. $41.9 \mathrm{nmol} / \mathrm{l}$ in winter) and the smallest in Poland $(67.1 \mathrm{nmol} / \mathrm{l}$ in summer $v$. $50.4 \mathrm{nmol} / \mathrm{l}$ in winter). Example calculation: on 20 May, the SSA reaches 0.5 , therefore the estimate for a participant in Germany would be $67.0+0.5 \times 25.1=79.6 \mathrm{nmol} / \mathrm{l}$. Horizontal lines indicate vitamin $\mathrm{D}$ status intervals: $<25 \mathrm{~nm}$ deficient, 25-50 nм insufficient, 50-75 nм sufficient, $>75 \mathrm{~nm}$ optimal range. 
Table 2. Association of 25-hydroxy vitamin $\mathrm{D}_{3}\left(25(\mathrm{OH}) \mathrm{D}_{3}\right)$ levels with the predictors 'study centre' and 'seasonal amplitude** (Coefficients with their standard errors)

\begin{tabular}{|c|c|c|c|c|c|c|c|}
\hline Locations & Predictor & $\begin{array}{l}\text { Coefficient } \\
(\mathrm{nmol} / \mathrm{l})\end{array}$ & $\begin{array}{c}\mathrm{SE} \\
(\mathrm{nmol} / \mathrm{l})\end{array}$ & $t$ Value & $P$ & $\begin{array}{l}\text { Seasonal minimum } \\
(\mathrm{nmol} / \mathrm{l})\end{array}$ & $\begin{array}{c}\text { Seasonal maximum } \\
(\mathrm{nmol} / \mathrm{l})\end{array}$ \\
\hline \multirow[t]{2}{*}{ Munich, Germany } & Study centre & $67 \cdot 0$ & 1.5 & $43 \cdot 6$ & $<0.001$ & 41.9 & $92 \cdot 1$ \\
\hline & Seasonal amplitude & $25 \cdot 1$ & 1.3 & $18 \cdot 8$ & $<0.001$ & & \\
\hline \multirow[t]{2}{*}{ Athens, Greece } & Study centre & 60.7 & 1.5 & 39.9 & $<0.001$ & $45 \cdot 6$ & $75 \cdot 8$ \\
\hline & Seasonal amplitude & $15 \cdot 1$ & 1.3 & 11.8 & $<0.001$ & & \\
\hline \multirow{2}{*}{ Dublin, Ireland } & Study centre & $57 \cdot 1$ & 1.4 & $40 \cdot 3$ & $<0.001$ & 38.0 & $76 \cdot 1$ \\
\hline & Seasonal amplitude & $19 \cdot 0$ & $1 \cdot 3$ & 14.7 & $<0.001$ & & \\
\hline \multirow{2}{*}{ Maastricht, The Netherlands } & Study centre & 73.0 & 1.4 & $52 \cdot 3$ & $<0.001$ & 63.0 & 83.1 \\
\hline & Seasonal amplitude & $10 \cdot 0$ & 1.1 & 8.9 & $<0.001$ & & \\
\hline \multirow[t]{2}{*}{ Warsaw, Poland } & Study centre & $58 \cdot 7$ & 1.5 & 39.5 & $<0.001$ & 50.4 & $67 \cdot 1$ \\
\hline & Seasonal amplitude & 8.4 & 1.2 & 6.7 & $<0.001$ & & \\
\hline \multirow[t]{2}{*}{ Navarra, Spain } & Study centre & $65 \cdot 2$ & 1.5 & $42 \cdot 2$ & $<0.001$ & $49 \cdot 9$ & $80 \cdot 4$ \\
\hline & Seasonal amplitude & $15 \cdot 2$ & 1.3 & 11.8 & $<0.001$ & & \\
\hline \multirow[t]{2}{*}{ Reading, UK } & Study centre & $58 \cdot 2$ & 1.5 & 38.2 & $<0.001$ & $42 \cdot 1$ & 74.2 \\
\hline & Seasonal amplitude & $16 \cdot 1$ & 1.4 & $11 \cdot 3$ & $<0.001$ & & \\
\hline
\end{tabular}

* The study centre and the season per study centre were very good predictors of $25(\mathrm{OH}) \mathrm{D}_{3}$ levels. In Dublin, for example, the model estimated that the $25(\mathrm{OH}) \mathrm{D}_{3}$ levels oscillate by an amplitude of $\pm 19.0 \mathrm{nmol} / \mathrm{l}$ around a mean of $57.1 \mathrm{nmol} / \mathrm{l}$ over the seasons. This translates to a minimum of $38.0 \mathrm{nmol} / \mathrm{l}$ on the $20 \mathrm{January}, 57.1 \mathrm{nmol} / \mathrm{l}$ on the $20 \mathrm{April}$ and a maximum of $76 \cdot 1 \mathrm{nmol} / \mathrm{l}$ on the 21 July.

was suitable for determination of status levels for vitamin $\mathrm{D}$, and thus for application in the Food4Me study.

In total, 3778 DBS samples were analysed: 453 from Germany, 560 from Greece, 554 from Ireland, 634 from The Netherlands, 530 from Poland, 555 from Spain and 492 from the UK. From 1003 participants, DBS at three time points could be measured, from 307 participants two time points could be measured, whereas for 155 participants only one time point could be measured. In addition, no samples were received for analysis from 259 participants $(19.7 \%$ of total cohort who completed the study); in addition, although a further sixty-seven samples gave valid results for $25(\mathrm{OH}) \mathrm{D}_{3}$ concentrations, they lacked a sampling date, and thus could not be included in the analysis of effects of seasons. Only eighty samples either did not meet the quality criteria or had insufficient blood spots for analysis of all the required parameters, and only five analyses failed due to technical errors. Overall, the quality of the samples was very good, showing that the methodology is suitable for unsupervised sampling at home following provision of detailed instructions to the participants including, for example, a video demonstration of the sampling process.

The data for $25(\mathrm{OH}) \mathrm{D}_{3}$ concentration are presented in Fig. 2. None of the samples contained $25(\mathrm{OH}) \mathrm{D}_{2}$ above the LOQ of $25 \mathrm{nmol} / 1$. The seasonality of the $25(\mathrm{OH}) \mathrm{D}_{3}$ status for the participants of each study centre is clearly visible $(P<0.001)$ with significantly higher concentrations in summer than in winter $(67 \cdot 1-92 \cdot 1 v$. 38.0-63.0 $\mathrm{nmol} / \mathrm{l})$. Details of the observed correlations are given in Table 2. Owing to the different starting dates, and the different duration of the study due to recruiting progress in each centre, the coverage of the annual time period varied between centres, but it usually included the seasonal minimum and maximum. DBS collection over more than 12 months occurred in only two centres - Dublin in Ireland and Reading in the UK. The values for the seasonal minima and maxima of $25(\mathrm{OH}) \mathrm{D}_{3}$ levels varied by country (Fig. 2). The largest seasonal changes were observed in the participants from Germany $92.1 \mathrm{nmol} / \mathrm{l}$ in summer $v$. $41.9 \mathrm{nmol} / \mathrm{l}$ in winter, whereas the smallest changes were recorded in Poland (67.1 $v$. $50.4 \mathrm{nmol} / 1$, respectively). Overall, the highest values were found in late summer (21 July), and the lowest values were found in late winter (20 January). This is in good agreement with data from the literature, although the number of reports from comparable cohorts is limited ${ }^{(27)}$.

To assess the performance of the analytical method and the statistical model, a leave-one-out cross-validation was performed (Fig. 3). Only participants for whom all three planned measurements were available were included in the validation. For each measured value, a prediction was performed by taking into account the site and the season as fixed factors and the subject ID as a random factor. The two other measurements for the same participant were included each time, and the measurement to be predicted was excluded each time. In the absence of a gold standard, these predicted values were considered as the most plausible reference values against which the actual measurement values could be compared. The resulting Pearson's correlation between measured and modelled values was $r 0.65$, and the differences between the modelled and measured values had an SD of $21.2 \mathrm{nmol} / \mathrm{l}$. This variation is attributable both to biological and analytical variation.

\section{Discussion}

With 3778 valid measurements obtained from 1465 participants, to the best of our knowledge, the Food4Me study is the largest study using DBS samples where the participants did the sampling themselves at home. Sample preparation was designed to be as fast and as easy as possible, with the potential to perform all necessary steps in a multiwell plate. A crucial step for the whole methodology was the calibration of the assay. Although analysis of $25(\mathrm{OH}) \mathrm{D}_{3}$ from DBS has been applied before, we introduced improvements including calibration using DBS with endogenous analyte content and corrected by the haematocrit content of the calibration samples. As $25(\mathrm{OH}) \mathrm{D}_{3}$ is an endogenous compound, no blank samples of blood were 

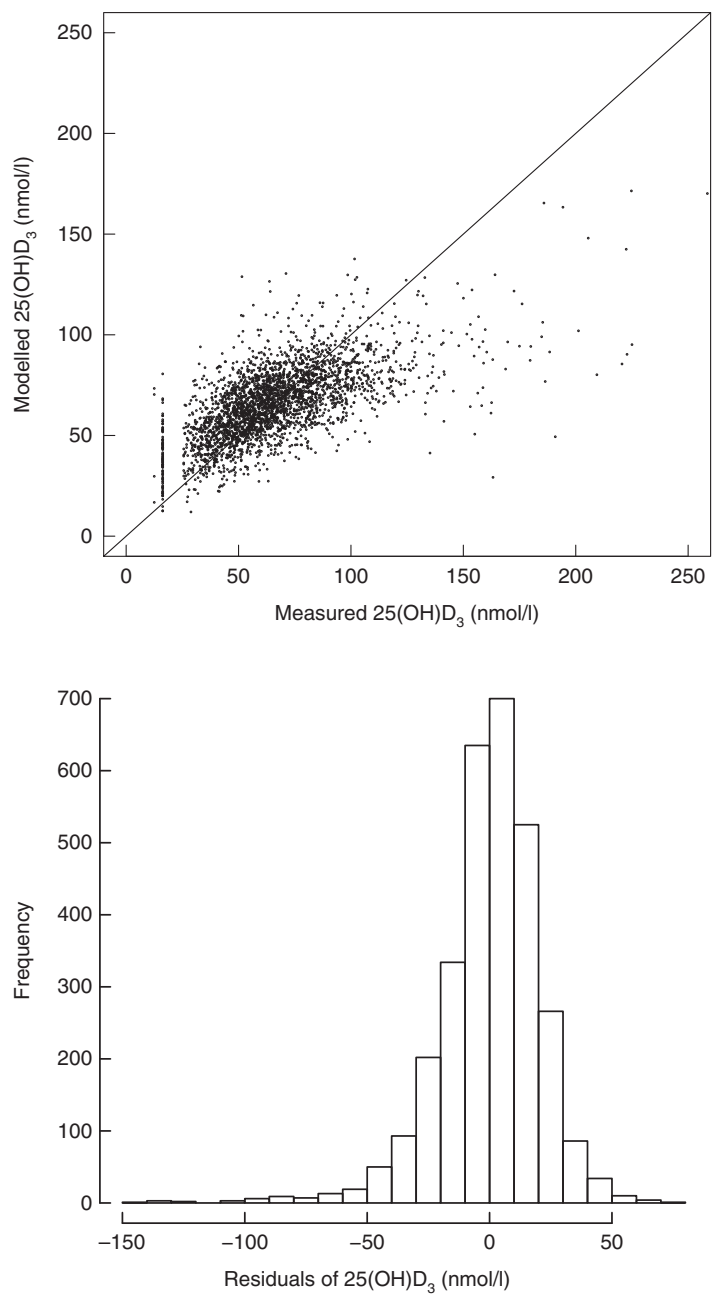

Fig. 3. Comparison between modelled 25-hydroxy vitamin $\mathrm{D}_{3}\left(25(\mathrm{OH}) \mathrm{D}_{3}\right)$ values and actually measured $25(\mathrm{OH}) \mathrm{D}_{3}$ values, based on a leave-one-out cross-validation of the model in Table 2 and Fig. 2. Only participants for whom all three pre-planned dried blood spots-based measurements were available were included in the validation. For each measured value, a prediction was performed by taking into account the site and the season as fixed factors and the subject ID as a random factor. Although the two other measurements of the same participant were included each time, the measurement to be predicted was excluded each time. The resulting Pearson's correlation between measured and modelled values was $r 0.65$, and the differences between the modelled and measured values had an SD of $21.2 \mathrm{nmol} / \mathrm{l}$.

available. In addition, spiking of blood with $25(\mathrm{OH}) \mathrm{D}_{3}$ was considered to be questionable, as the endogenous analyte is almost completely protein bound, and conditions to simulate this with spiking were difficult to achieve. Using blood with endogenous $25(\mathrm{OH}) \mathrm{D}_{3}$ content simplifies the calibration procedure and makes the methodology more robust as unknowns and calibration samples consist of the same matrix. As reference values for many micronutrients including vitamin D are typically reported as plasma levels, a conversion from whole blood is needed. This conversion is based on the haematocrit content that varies from person to person ${ }^{(28)}$. As was the case in the Food4Me study, this value is often not available for study samples. Different approaches to circumvent this issue have been proposed $^{(29)}$, which typically require an additional measurement of a second blood component such as $\mathrm{K}^{(30)}$, for normalisation, an approach that introduces additional analytical variance. For the present study, a haematocrit value of $41.5 \%$ was assigned to samples from female subjects and a value of $46.5 \%$ to samples from male subjects. As the study design did not include the concurrent sampling of venous blood and the analysis for $25(\mathrm{OH}) \mathrm{D}_{3}$ in the resulting plasma as a reference, the influence of haematocrit on the accuracy of the results could not be verified independently. However, we estimated that our assumption introduced a small amount of additional variation, perhaps $<10 \%$ - that is, when considering an exemplified normal range of $36-47 \%$ haematocrit for women, a plasma concentration of $50 \mathrm{nmol} / \mathrm{l}$ calculated for a mean of $41.5 \%$ haematocrit could vary between 45.7 and $55.2 \mathrm{nmol} / 1$. The influence of other factors including spot size and location of punches on vitamin D determination in DBS have been reported in the literature ${ }^{(31)}$. Although our new method did not reach the accuracy of the reference analytics of $25(\mathrm{OH}) \mathrm{D}_{3}$ from plasma by LC-MS/MS, the results are comparable with, or better than, those reported for other DBS-based methods - for example, accuracy by recovery was reported in the range of $95.2-102.7 \%$ (four concentration levels) $^{(12)}$ and $80-118 \%$ (three levels) $^{(9)}$ and intra-assay precision in the range of $3 \cdot 2-6 \cdot 9^{(12)}, 8-13^{(9)}$ and $11-13 \%$ relative standard deviation (RSD) (three levels, inter-assay $)^{(11)}$. Overall, the new method was found to be suitable for status-level determination, as indicated by the performance criteria in comparison with international guidelines.

In the Food4Me study, sampling of the blood from the fingertip by participants was carried out unsupervised. This posed questions of compliance with the sampling protocol, and with regard to the impact of deviations from the protocol on overall sample quality. We found that the large majority of blood spots were of very good quality when assessed visually compared with our quality control criteria. This also suggests that this finger-prick approach to blood collection was well accepted by the participants, given the total of acceptable measurements, and that provision of both written and video instruction ensures that blood samples can be obtained reliably from untrained participants in large cohort studies across multiple countries. Quality aspects not assessable by visual inspection include too long or too short drying times of the blood and excessive sun or heat exposure of the sample before it reached the research centre. In addition, the identity of the blood donor could not be verified independently, although an embedded validation study of $10 \%$ of participants was carried out, which included verification of identity based on genotype-provided reassurance on this question ${ }^{(32)}$. Therefore, the consistency of data points for each individual participant was used as an indirect quality indicator. The resultant sequential assessment of vitamin D status from three DBS measurements over 6 months is unique. A rapid increase in vitamin D status is possible in case of extended sun exposure or the use of high-dose supplementation, but a fast decline is very unlikely, because $25(\mathrm{OH}) \mathrm{D}_{3}$ has a half-life of approximately $20 \mathrm{~d}$ following oral administration ${ }^{(33)}$. The demonstrated consistency of consecutive data from the individual participants proves the robustness of the methodology and the suitability of the DBS approach with unsupervised sampling for status determination of vitamin $\mathrm{D}_{3}$. 
As expected, the results for all countries showed a strong seasonality with lowest mean vitamin $\mathrm{D}_{3}$ status levels observed towards the end of January and highest mean status levels observed towards the end of July. Although representative data for vitamin D status is available in some European countries ${ }^{(27)}$, data on seasonality are more limited. For the UK, lowest level of vitamin $\mathrm{D}_{3}$ concentrations were reported in February and the highest levels in September during the 2002-2004 period $^{(34)}$, with a difference of approximately $35 \mathrm{nmol} / \mathrm{l}$. Furthermore, reports for Germany ${ }^{(35,36)}$ clearly demonstrate a seasonality with the highest median value in June and the lowest median value in March with a difference of $23.4 \mathrm{nmol} / 1$ for $1998^{(36)}$. Although the participants of the present study may not be representative of each country, our results are comparable with the published data. An in-depth analysis of differences between countries will be carried out separately.

Overall, the present study provided valuable insight into effects of seasonality of vitamin $\mathrm{D}_{3}$ status in 1465 participants from seven European countries. The DBS-based methodology of unsupervised sampling of DBS by the participants at home was found to be suitable for status determination of $25(\mathrm{OH}) \mathrm{D}_{3}$ in the setting of the large, international nutritional study Food4Me. This encourages application in future studies, and has the potential for simultaneous determination of other micronutrients from the same DBS samples.

\section{Acknowledgements}

This project was supported by the European Commission under the Food, Agriculture, Fisheries and Biotechnology Theme of the 7th Framework Programme for Research and Technological Development, grant number 265494.

The authors' contributions are as follows: U. H. drafted the manuscript and developed the analytical method together with M. B. who carried out all the sample measurements; F. F. R. performed the statistical analysis; P. W. contributed to the design of the analytical methodology and data interpretation, J. C. M. was the study director of the proof-of-principle study of Food4Me; H. D., M. G., J. A. L., Y. M., J. A. M., W. H. M. S. and I. T. contributed to the design of the proof-of-principle study and were principle investigator for their respective research centre; L. B., R. F., H. F., E. R. G., M. G., K. H., S. K., C. P. L., K. M. L., A. L. M., C. F. M. M., C. C.-M., G. M., S. N.-C., C. B. O.'D., R. S.-C., A. S., L. T., M. C. W. and C. W. contributed to the study design and execution at the research centres. All authors contributed to, read and approved the final version of the manuscript.

U. H., M. B., F. F. R. and P. W. are employed by DSM Nutritional Products. The other authors have no potential financial or personal conflicts of interest to declare.

\section{References}

1. Demirev PA (2013) Dried blood spots: analysis and applications. Anal Chem 85, 779-789.

2. McDade TW, Williams S \& Snodgrass JJ (2007) What a drop can do: dried blood spots as a minimally invasive method for integrating biomarkers into population-based research. Demography 44, 899-925.

3. Celis-Morales C, Livingstone K, Marsaux CM, et al. (2014) Design and baseline characteristics of the Food4Me study: a web-based randomised controlled trial of personalised nutrition in seven European countries. Genes Nutr 10, 1-13.

4. Guthrie R \& Susi A (1963) A simple phenylalanine method for detecting phenylketonuria in large populations of newborn infants. Pediatrics 32, 338-343.

5. Tanna S \& Lawson G (2011) Analytical methods used in conjunction with dried blood spots. Anal Methods 3, 1709-1718.

6. Shi H, Ma Y, Humphrey JH, et al. (1995) Determination of vitamin A in dried human blood spots by high-performance capillary electrophoresis with laser-excited fluorescence detection. J Chromatogr B Biomed Appl 665, 89-96.

7. Craft NE, Haitema T, Brindle LK, et al. (2000) Retinol analysis in dried blood spots by HPLC. J Nutr 130, 882-885.

8. O'Broin SD \& Kelleher BP (2008) A dried serum spot assay for vitamin $\mathrm{B}_{12}$. Clin Chem Lab Med 46, 354-358.

9. Eyles D, Anderson C, Ko P, et al. (2009) A sensitive LC/MS/MS assay of $25 \mathrm{OH}$ vitamin $\mathrm{D}_{3}$ and $25 \mathrm{OH}$ vitamin $\mathrm{D}_{2}$ in dried blood spots. Clin Chim Acta 403, 145-151.

10. Eyles DW, Morley R, Anderson C, et al. (2010) The utility of neonatal dried blood spots for the assessment of neonatal vitamin D status. Paediatr Perinat Epidemiol 24, 303-308.

11. Newman MS, Brandon TR, Groves MN, et al. (2009) A liquid chromatography/tandem mass spectrometry method for determination of 25-hydroxy vitamin $\mathrm{D}_{2}$ and 25-hydroxy vitamin $\mathrm{D}_{3}$ in dried blood spots: a potential adjunct to diabetes and cardiometabolic risk screening. J Diabetes Sci Technol 3, 156-162.

12. Higashi T, Suzuki M, Hanai J, et al. (2011) A specific LC/ ESI-MS/MS method for determination of 25-hydroxyvitamin $\mathrm{D}_{3}$ in neonatal dried blood spots containing a potential interfering metabolite, 3-epi-25-hydroxyvitamin $\mathrm{D}_{3}$.J Sep Sci 34, $725-732$.

13. Larkin EK, Gebretsadik T, Koestner N, et al. (2011) Agreement of blood spot card measurements of vitamin D levels with serum, whole blood specimen types and a dietary recall instrument. PLOS ONE 6, e16602.

14. Motohara K, Endo F \& Matsuda I (1987) Screening for late neonatal vitamin $\mathrm{K}$ deficiency by acarboxyprothrombin in dried blood spots. Arch Dis Child 62, 370-375.

15. O'Broin S \& Gunter E (2002) Dried-serum spot assay for folate. Clin Chem 48, 1128-1130.

16. Erhardt JG, Craft NE, Heinrich F, et al. (2002) Rapid and simple measurement of retinol in human dried whole blood spots. J Nutr 132, 318-321.

17. Bikle DD, Gee E, Halloran B, et al. (1986) Assessment of the free fraction of 25-hydroxyvitamin $\mathrm{D}$ in serum and its regulation by albumin and the vitamin D-binding protein. I Clin Endocrinol Metab 63, 954-959.

18. Al-oanzi ZH, Tuck SP, Raj N, et al. (2006) Assessment of vitamin D status in male osteoporosis. Clin Chem $\mathbf{5 2}$, $248-254$.

19. Livingstone KM, Celis-Morales C, Navas-Carretero S, et al. (2015) Profile of European adults interested in internet-based personalised nutrition: the Food4Me study. Eur J Nutr (epublication ahead of print version 17 April 2015).

20. Lauridsen C, Halekoh U, Larsen T, et al. (2010) Reproductive performance and bone status markers of gilts and lactating sows supplemented with two different forms of vitamin D. J Anim Sci 88, 202-213.

21. European Medicines Agency (2011) Guideline on bioanalytical method validation. EMEA/CHMP/EWP/192217/2009. London: European Medicines Agency. 
22. Timmerman P, White S, Globig S, et al. (2011) EBF recommendation on the validation of bioanalytical methods for dried blood spots. Bioanalysis 3, 1567-1575.

23. Bendik I, Friedel A, Roos FF, et al. (2014) Vitamin D: a critical and essential micronutrient for human health. Front Physiol 5, 248.

24. Bischoff-Ferrari H, Dawson-Hughes B, Stocklin E, et al. (2012) Oral supplementation with $25(\mathrm{OH}) \mathrm{D}_{3}$ versus vitamin $\mathrm{D}_{3}$ : effects on $25(\mathrm{OH}) \mathrm{D}$ levels, lower extremity function, blood pressure, and markers of innate immunity. J Bone Miner Res 27, 160-169.

25. R Core Team (2013) R: A Language and Environment for Statistical Computing. Vienna, Austria: R Foundation for Statistical Computing. http://www.R-project.org/

26. Pinheiro J, Bates D, DebRoy S, et al. (2014) nlme: Linear and Nonlinear Mixed Effects Models. R package version 3.1-117. http://CRAN.R-project.org/package =nlme

27. Wahl DA, Cooper C, Ebeling PR, et al. (2012) A global representation of vitamin $\mathrm{D}$ status in healthy populations. Arch Osteoporos 7, 155-172.

28. Thirup P (2003) Haematocrit: within-subject and seasonal variation. Sports Med 33, 231-243.

29. De Kesel PM, Sadones N, Capiau S, et al. (2013) Hemato-critical issues in quantitative analysis of dried blood spots: challenges and solutions. Bioanalysis 5, 2023-2041.

30. Capiau S, Stove VV, Lambert WE, et al. (2013) Prediction of the hematocrit of dried blood spots via potassium measurement on a routine clinical chemistry analyzer. Anal Chem 85 404-410.

31. Kvaskoff D, Ko P, Simila HA, et al. (2012) Distribution of 25-hydroxyvitamin $\mathrm{D}_{3}$ in dried blood spots and implications for its quantitation by tandem mass spectrometry. J Chromatogr B Analyt Technol Biomed Life Sci 901, $47-52$.

32. Celis-Morales C, Livingstone KM, Woolhead C, et al. (2015) How reliable is internet-based self-reported identity, socio-demographic and obesity measures in European adults? Genes Nutr 10, 476.

33. Smith JE \& Goodman DS (1971) Turnover and transport of vitamin D and of a polar metabolite with the properties of 25 -hydroxycholecalciferol in human plasma. J Clin Invest $5 \mathbf{5 0}$ 2159-2167.

34. Hypponen E \& Power C (2007) Hypovitaminosis D in British adults at age $45 \mathrm{y}$ : nationwide cohort study of dietary and lifestyle predictors. Am J Clin Nutr 85, 860-868.

35. Zittermann A, Scheld K \& Stehle P (1998) Seasonal variations in vitamin $\mathrm{D}$ status and calcium absorption do not influence bone turnover in young women. Eur J Clin Nutr $\mathbf{5 2}$, 501-506.

36. Hintzpeter B, Mensink GBM, Thierfelder W, et al. (2008) Vitamin D status and health correlates among German adults. Eur J Clin Nutr 62, 1079-1089. 